\section{Crystallography and NMR: An Overview}

\author{
Robin K. Harris \\ University of Durham, Durham City, UK
}

1 Introduction

2 Limitations of Diffraction Techniques

3 NMR and its Crystallographic Significance

5 Concluding Remarks

6 Related Articles

7 End Notes

8 References
4 Required Information
1

2

4

4

11

11

11

12

\section{INTRODUCTION}

Although there is no problem defining nuclear magnetic resonance (NMR), different people interpret the term crystallography differently. In particular, experts in diffraction studies of crystals frequently use the term as though it is synonymous with structural information obtained by diffraction methods. However, historically, crystals were studied and classified by their morphology alone. It became recognized, for example, that different habits can result from the same underlying symmetry.

The term crystallography is derived from the Greek words crystallon, meaning cold drop/frozen drop, and graphein, meaning "write". It refers to the experimental science of determining the arrangement of atoms in solids, specifically for what are now called crystalline solids. In older usage, it is simply the scientific study of crystals. The use of the words "atoms in solids" implies that amorphous solids may be included in "crystallography", but this is contentious and such systems will not, in general, be dealt with here. Moreover, the original usage of the word (dating from eighteenth century Latin "crystallographiæ" and French "cristallographie") referred to the external shape of crystals (morphology) rather than the internal structure, and this is still included in the normal meaning. Dictionaries define "crystallography" in various ways. Some include both internal arrangement and external morphology of crystals. Others discuss the properties of crystals and/or their classification. One of the simplest definitions states merely "the study of crystal form and structure". ${ }^{1}$ Most dictionaries (as opposed to encyclopedias) do not mention diffraction in the definition.

Of course, studies of morphology alone tend to obscure the varied nature of the internal arrangements. A student first meeting crystallographic concepts may be lead to believe in crystalline perfection, i.e., the existence of perfect threedimensional translational symmetry of a unit cell with fixed contents, belonging to a well-defined space group. Reality shows a greater variety of possibilities within the general idea of "crystallinity", questions of nonstoichiometric systems, disorder, defects, incommensurate cases, and molecular-level mobility all complicating the general situation. Moreover, the surface structure of crystals deviates from that of their bulk contents; surface structures are extremely important, since they govern interactions with the surrounding medium, as in all types of composite materials.

These facts clearly indicate the width of usage and applicability of the terms crystallography and crystal structure. As a result, many tools must be brought to fully understand both the macroscopic nature and atomic-level structure of crystalline materials. Over the last century, diffraction experiments have emerged as the primary way of determining the disposition of atoms in unit cells of crystals. When a new compound is synthesized and obtained in crystalline form, it is usually of high priority to determine its solid-state structure by means of single-crystal diffraction studies. Indeed, this is also frequently the key way of obtaining the molecular structure definitively for organic and organometallic compounds, though solution-state NMR is equally powerful for such determinations. Indeed, synthetic chemists are often not interested in crystallographic details other than the molecular structure. Single-crystal diffraction work is accepted as the "gold standard" for providing molecular structure (and the structure of network systems) for crystalline systems. It will undoubtedly remain so.

However, NMR has much to offer the crystallographer because it can both supplement and complement the diffraction results. The term NMR crystallography has come into common use in the last decade, though some diffraction experts decry it. ${ }^{a}$ Historically, NMR has given crystallographic information since the earliest days of the technique. It was recognized, for example, that dipolar interactions could yield direct information on internuclear distances. This can be achieved easily when there are isolated pairs of protons in a crystal. In 1948 Pake $^{2}$ reported the line-pair separations of the water proton resonances for a single crystal of calcium sulfate dihydrate (gypsum), $\mathrm{CaSO}_{4} \cdot 2 \mathrm{H}_{2} \mathrm{O}$, as a function of the crystal orientation in $\mathbf{B}_{0}$ (Figure 1). Thence, he derived the internuclear distance between the protons in the hydrate water molecules as $1.58 \AA$. This was at a time (1948) when detecting hydrogen atoms by X-ray diffraction was problematic (as it remained for many years - and even today this can cause difficulties in some cases). Pake also illustrated the lineshape for the proton spectrum of a polycrystalline sample of gypsum, which is of the type now well known as a Pake doublet, allowing internuclear distance information to be obtained even in the absence of a single crystal. An article a year later ${ }^{3}$ illustrates the experimental powder pattern for $\mathrm{ClH}_{2} \mathrm{C}-\mathrm{CH}_{2} \mathrm{Cl}$ (Figure 2), together with a simulated Pake doublet (though this case does not really have isolated proton spin pairs). The same paper states the following in the introduction: "The development of experimental methods for observing nuclear magnetism has provided a means of investigating certain aspects of molecular and crystal structure" (italicization inserted here).

Today, NMR methods (and consequently the nature of results) have advanced enormously so that it is now feasible to solve crystal structures for polycrystalline samples, in favorable cases, by NMR means, given only the unit cell parameters and space group from diffraction work, ${ }^{4}$ and to obtain structural information about crystalline proteins. ${ }^{5}$ While no one would suggest that NMR will ever replace diffraction work, it can and does address crystallographic matters that diffraction methods struggle with. The complementarity of the 


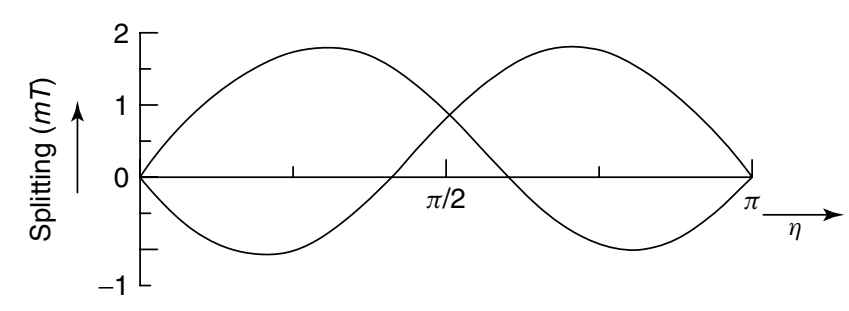

Figure 1 Proton line-pair separations for a single crystal of gypsum as a function of the angle, $\eta$, between the [100] direction and the applied magnetic field

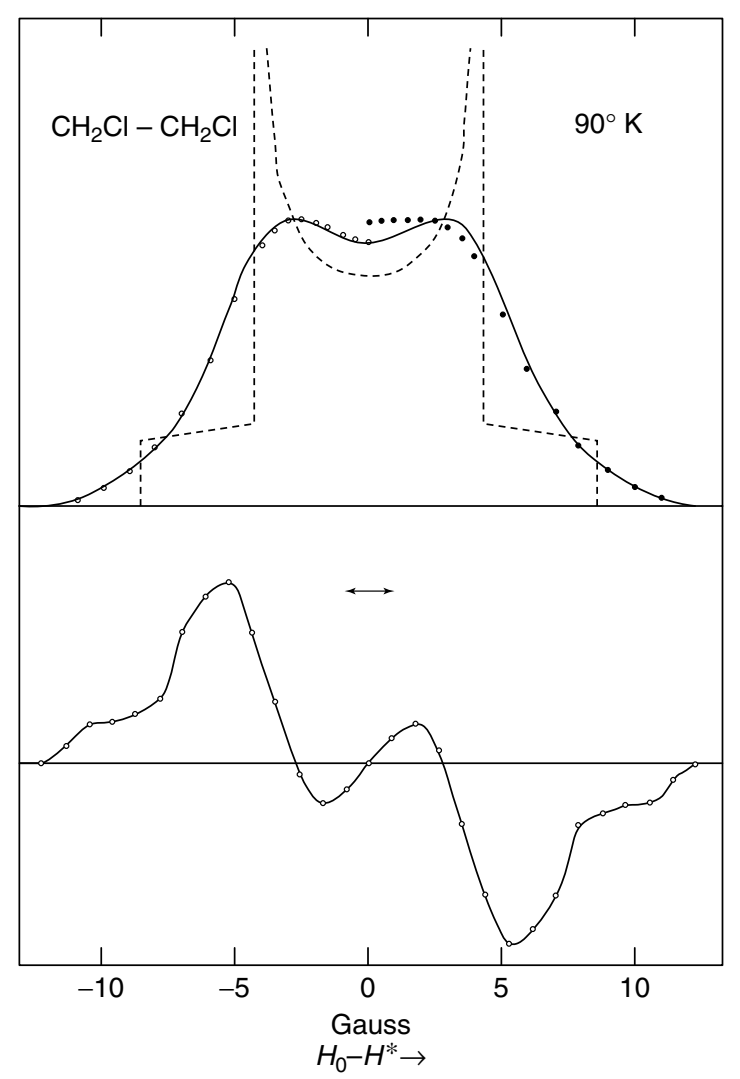

Figure 2 The proton magnetic resonance absorption line and its derivative for solid 1,2-dichloroethane at $90 \mathrm{~K}$. The solid lines in both parts of the figure (the lower part being in derivative mode) represent experimental data. In the upper part of the figure, the open circles in the left half are theoretical values computed for a proton separation of $1.70 \AA$ and the points in the right half are for $1.72 \AA$. The dashed curve is the theoretical lineshape for a crystal powder with a proton separation of $1.70 \AA$ and in which magnetic interactions are confined to pairs of nuclei. The upper diagram illustrates the well-known "Pake doublet". (Adapted with permission from Ref. 3, ㄷ American Institute of Physics, 1949)

two techniques is based on the fact that diffraction methods rely on the presence of long-range order, whereas NMR spectra reflect the local environment of the nucleus under study. This means that a combination of the two techniques is especially powerful (see Interplay between Solid-State NMR and SingleCrystal X-Ray Diffraction; Crystal Structure Determination by the Combined Use of NMR and Powder Diffraction). For instance, NMR can give structural information on imperfect crystalline situations (such as are mentioned above) and on crystal surfaces-all cases for which diffraction methods struggle to give definitive data. Indeed, the NMR techniques are not limited to crystalline materials; hence, information on molecular-level structure and local environment can be obtained for amorphous solids (and for heterogeneous systems) for which diffraction is largely inapplicable. Such systems provide important applications of NMR, but the present article is limited to crystalline materials (except for one case).

NMR work on single crystals is feasible (and yields detailed crystallographic information) ${ }^{\mathbf{6}}$ (see Chemical Shift Tensors in Single Crystals). However, rather large crystals and special probes are required; hence, single-crystal NMR is not common (though more use is perhaps justified). Polycrystalline samples are normally studied, usually using magic-angle spinning. Fortunately, given the range of specialist pulse sequences available for deriving specific pieces of information, the loss of information incurred by not using single crystals is, in general, rather small. The remainder of this article will only involve polycrystalline systems.

One of the simplest uses of solid-state NMR in crystallography is to distinguish between habit (samples giving identical MAS spectra) and polymorphism (spectra being different) ${ }^{7}$ (see Polymorphism \& Solvate Formation: Organic and Pharmaceutical Systems; Polymorphism and Related Phenomena). Polymorphism is ubiquitous throughout chemistry and its study is essential to many solid-state applications, especially in the pharmaceutical industry. Distinctions between polymorphs by NMR are generally clear-cut and are rendered particularly straightforward by taking advantage of the multinuclear nature of NMR. For example, ${ }^{13} \mathrm{C}$ spectra may reveal differences between samples that are not apparent from ${ }^{15} \mathrm{~N}$ studies. The distinctions enable studies of phase transitions to be made by NMR (see Structural Phase Transitions). Another simple use of solid-state NMR is in detecting isomorphism. Thus, Figure 3 shows $^{8}$ that four samples of finasteride (see I) solvates are isomorphous while the remaining (acetic acid) one has a different structure. This example also demonstrates the superb direct chemical information given by NMR, i.e., the signals definitively determine the solvent molecules involved, in contrast to diffraction that, because of solvent molecule mobility in the solvates, can only show a volume of electron density of indiscriminate nature.

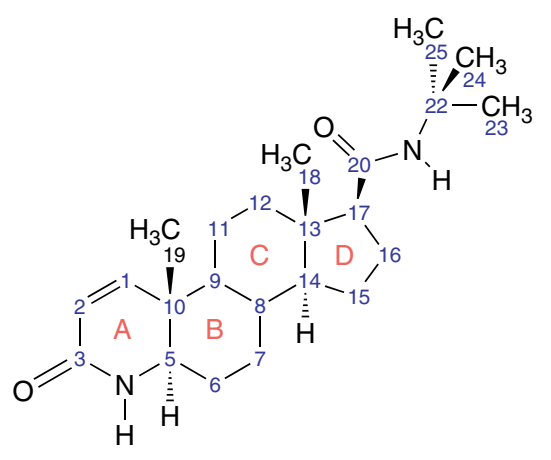

Finasteride, I

\section{LIMITATIONS OF DIFFRACTION TECHNIQUES}

Although it is stressed that the superb ability of diffraction techniques to obtain structural information for crystalline 


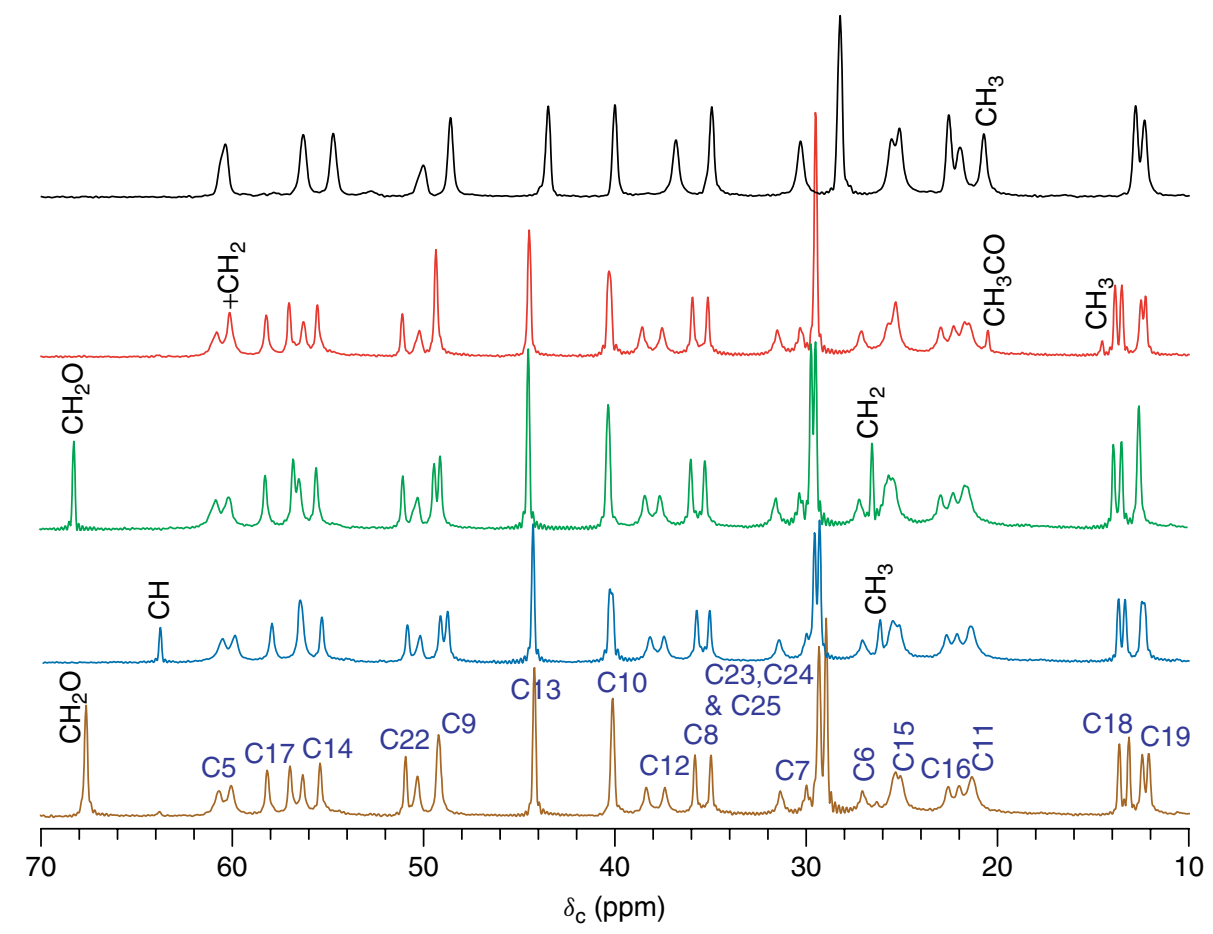

Figure 3 Carbon-13 CPMAS spectra of five solvates of finasteride (I). Top to bottom: acetic acid solvate; ethyl acetate solvate hydrate; THF solvate hydrate; isopropanol solvate hydrate; and dioxane solvate hydrate. Assignments are indicated for the solvent peaks. (Reproduced with permission from Ref. 8, (c) 2007, John Wiley \& Sons Ltd.)

materials (most importantly, the positions of atoms in the unit cell) is and will remain unsurpassed, there are certain inherent difficulties with obtaining some facets of crystallographic information from single-crystal diffraction experiments. These can be summarized as follows:

- For the best results, single crystals are required, which should be of the order of $0.001 \mathrm{~mm}^{3}$ or greater (though with synchrotron radiation much smaller crystals can be effectively studied).

- The crystals need to be as perfect as possible, i.e., without substantial defects-though special techniques can cope with some imperfections (such as twinning).

- Disorder, either spatial or temporal, causes uncertainties.

- For X-ray diffraction (though not neutron diffraction), distinctions between atoms of different elements with similar atomic numbers are sometimes difficult to make (especially in isoelectronic cases).

- Historically, hydrogen atoms have been difficult to locate with accuracy, especially in the presence of heavy atoms.

- The crystal studied may not be representative of the bulk sample (though powder diffraction experiments can be used to clarify this matter).

- Amorphous solids cannot be adequately examined.

Advances in technology are rendering some of these difficulties less restrictive. In addition, in recent years, the power of powder diffraction techniques has been greatly increased so that it is feasible in many cases to obtain detailed crystal structures by this means. ${ }^{\mathbf{9}}$ This is particularly valuable, for example, for samples of small crystals embedded in an amorphous matrix, as is the case for many ceramic systems. Other advances in X-ray diffraction procedures allow electron distributions to be modeled in detail, and the location of hydrogen atoms is much improved. However, the fact remains that $\mathrm{X}$ rays locate electron density, not nuclei, though neutron diffraction does depend on the positions of atomic nuclei.

There are two major areas in which complementary information is desirable:

1. Molecular-level mobility: Most X-ray diffraction measurements that result in structure determinations now take place at $120 \mathrm{~K}$. However, even at this temperature, there is significant mobility over the timescale of a typical diffraction experiment (generally ca. 4-6h). Analysis of diffraction patterns can take some account of this (especially for vibrational motion) by using ellipsoids based on atomic displacement factors, though for X-ray measurements these actually depict electron density distributions. Site exchange (temporal disorder) by rapid jump motions can be accounted for by the use of fractional populations if the sites are distinguishable. However, when equivalent sites are involved, as with $120^{\circ}$ rotations of tertiary butyl groups, the exchange cannot be easily detected by diffraction methods. Moreover, obtaining information on the kinetics of molecular-level motion is extremely difficult by diffraction techniques. In this context, most vibrations occur at a sufficiently high frequency that their effects on both diffraction patterns and NMR spectra can be ignored.

2. Purely spatial (i.e., static) disorder raises a different problem, in that, in principle, the structure is not properly spatially repetitive; hence, it is not possible in strict theory to define a space group except on an average basis. In such cases, the analysis of diffraction patterns results in structures with fractional occupations. Differently ordered domains are difficult to investigate. 


\section{NMR AND ITS CRYSTALLOGRAPHIC SIGNIFICANCE}

The relationship of NMR to crystallography is a complex one (see Crystallography by NMR: Fundamental Concepts). NMR parameters can provide substantial crystallographic information in both direct and indirect ways. Such information derives from the various NMR measurables (see Tensors in NMR; Measurements on Static Samples \& via Spinning Sideband Analysis), namely, the following:

- Isotropic chemical shifts (see Magnetic Shielding and Chemical Shifts: Basics; Chemical Shifts \& Solid-State Structure): Traditionally, these have been used in the solution state to give direct information on molecular-level chemical structure. They can be used in similar manner for crystalline solids. ${ }^{\mathbf{1 0}}$ They can also define the number of nuclei in different environments for relevant nuclei in the crystal structure. This will determine the crystallographic asymmetric unit. In favorable circumstances, molecular symmetry and even space group information are obtained. In addition, chemical shifts often provide knowledge about intermolecular interactions, for example, the location of hydrogen bonds.

- Full information on chemical shift tensors: The anisotropy and asymmetry of the tensor (see Chemical Shift Tensor Measurement in Solids) relate directly to the detailed electronic environment of the atom concerned and hence correlate with the information obtainable from X-ray diffraction (see Chemical Shift Anisotropy and Asymmetry: Relationships to Crystal Structure).

- Dipolar coupling (see Dipolar and Indirect Coupling: Basics): This spin-spin interaction gives interatomic distance information directly and provides a very powerful method of studying crystal structure. However, molecularlevel motion can strongly affect the observed dipolar coupling constants (see Dipolar Coupling: Molecular-Level Mobility) so that care needs to be taken in interpretation. When MAS is used, recoupling pulse sequences are frequently necessary to obtain dipolar coupling constants (see Dipolar Recoupling: Heteronuclear; Dipolar Recoupling: Homonuclear Experiments).

- Isotropic indirect spin-spin coupling ${ }^{\mathrm{b}}$ (see Dipolar and Indirect Coupling: Basics; Indirect Coupling and Connectivity): This parameter gives information largely on conformational matters and is especially powerful for studies of molecular structure within crystals. However, its utility for solids has hitherto been muted because splitting patterns are often obscured by the linewidths in solids, particularly for $(\mathrm{H}, \mathrm{H})$ and long-range $(\mathrm{C}, \mathrm{H})$ coupling. On the other hand, coupling involving heavier atoms can be informative. Moreover, sophisticated measurements can reveal coupling across hydrogen bonds, ${ }^{\mathbf{1 1}}$ giving intermolecular relationships in crystals (see Hydrogen Bonding in Crystalline Organic Solids).

- Quadrupolar coupling: This interaction depends on electric field gradients (EFGs) at the nuclei, which in turn depend sensitively on the local environment. The anisotropy and asymmetry of the coupling provide additional information related to the site symmetry of the relevant nuclei in crystalline materials (see Quadrupole Coupling: Applications to Crystallography).

- Relaxation times: Three such processes are of particular importance for obtaining information on molecular-level motion (see Dipolar Coupling: Molecular-Level Mobility; Rotational and Translational Dynamics). These are (a) spin-lattice (otherwise known as longitudinal) relaxation, with characteristic time designated $T_{1}$; (b) spin-lattice relaxation in the rotating frame, designated time $T_{1 \rho}$; and (c) spin-spin (otherwise known as transverse) relaxation, with time $T_{2}$. They have characteristic motional dependencies at rates of tens to hundreds of megahertz, tens of kilohertz, and low frequencies, respectively. The related phenomenon of spin diffusion also carries information about the solid state (see Spin Diffusion in Crystalline Solids).

Other articles will explore these parameters in considerable detail. In this one, examples will be quoted to give the flavor of "NMR crystallography". The derived information can facilitate the complete structure determination from singlecrystal or powder diffraction patterns. In many cases, the knowledge obtained is additional to that found from diffraction work. Applications of NMR crystallography have been made to biochemistry (see NMR Crystallography: Applications to Biochemistry), geochemistry (see Crystallography \& NMR: Applications to Geochemistry), organic and pharmaceutical chemistry (see Crystallography \& NMR: Applications to Organic \& Pharmaceutical Chemistry), and inorganic and materials chemistry (see Crystallography \& NMR: Applications to Inorganic \& Materials Chemistry).

\section{REQUIRED INFORMATION}

So what constitutes crystallographic information?

\subsection{Molecular-Level Information}

As mentioned above, one key aspect is the atomic bonding (connectivity) pattern, forming the molecules or networks. The long history of solution-state NMR in this context, predating most high-resolution solid-state NMR, indicated the potential of the technique for solids. That potential is now increasingly being fulfilled, especially by the use of isotropic chemical shifts. One aspect of importance lies in the fact that solidstate structures may not be identical to those in solution. A relatively early NMR example of this phenomenon was provided ${ }^{\mathbf{1 2}}$ by $\mathrm{Pt}\left(\mathrm{PPh}_{3}\right)_{2}\left(\mathrm{P}(\right.$ mesityl $\left.)=\mathrm{CPh}_{2}\right)($ II $)$. Phosphorus31 CPMAS spectra of the solid showed unambiguously (from chemical shifts) that this compound has an $\eta^{1}$-coordinated (see IIa) phosphaalkene ligand, in disagreement with the $\eta^{2}$ case (see IIb) indicated by NMR for the solution state. The CPMAS work confirmed single-crystal diffraction results, thus giving an example of solid-state NMR acting as a bridge between solution-state NMR and diffraction techniques.

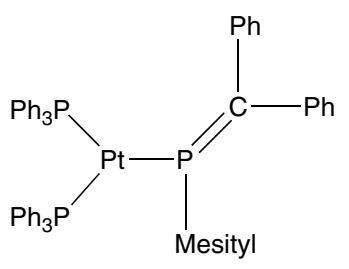

IIa

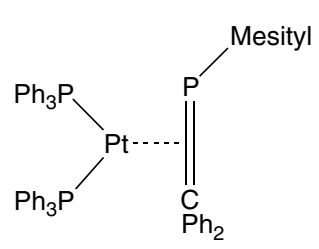

IIb 


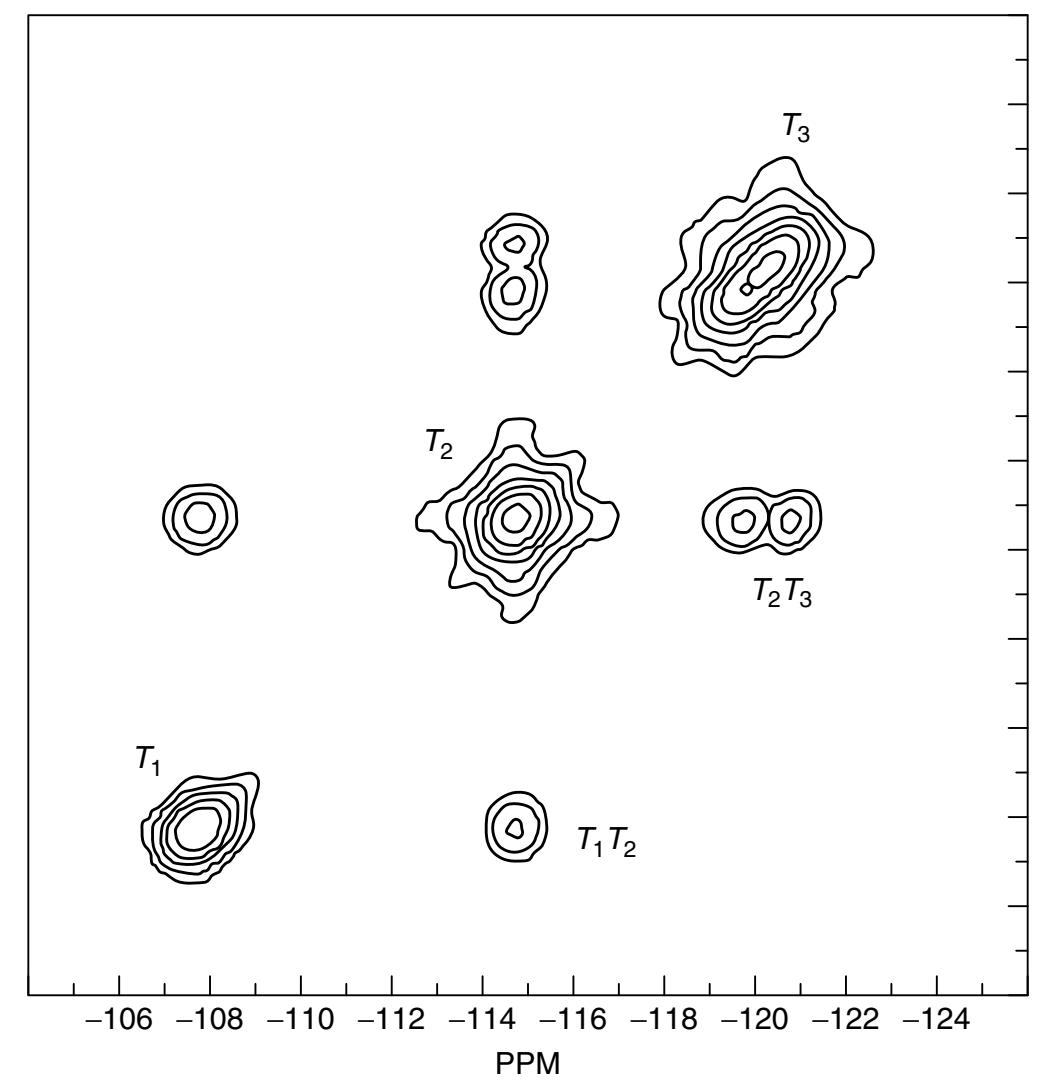

Figure 4 A two-dimensional homonuclear Si,Si connectivity plot for the zeolite ZSM-39, showing the connectivity (via oxygen) between the four different silicon sites. (Reprinted with permission from Ref. 13, () 1989 American Chemical Society)

CPMAS NMR provides an easier route to molecular structure in solids than diffraction because the link between isotropic chemical shifts and bonding environments is direct and specific to each atom. The correlations between shifts and bonding have been exhaustively worked out over five decades from solution-state NMR data. Indeed, solutionstate NMR is usually the preferred route to obtain molecular structure. Solid-state molecular-level structure determination can make use of the shift/structure correlations, but due regard has to be paid to specifically solid-state shift effects.

Indirect coupling can also play an important role in determining bonding patterns in crystalline solids (see Indirect Coupling and Connectivity), though, in contrast to solution-state work, this has mostly not involved $(\mathrm{H}, \mathrm{H})$ or $(\mathrm{C}, \mathrm{H})$ coupling. An early example of such usage was the determination ${ }^{13}$ of the connectivity in the zeolite ZSM39 via ${ }^{29} \mathrm{Si}$ COSY experiments (Figure 4). Recent pulse sequence developments ${ }^{14}$ have extended the scope for such determinations via $J$ coupling. Combinations of NMR experiments are sometimes necessary to provide full topological information on crystal structures. ${ }^{15}$ Dipolar coupling can also be of assistance in determining molecular structure in crystalline materials, though it has to be remembered that, because it occurs through space, it does not, per se, distinguish between connectivity and intermolecular proximity.

Of course, there is more to molecular structure than connectivity. In particular, conformations of flexible chains are of importance - and these can be expected to differ between

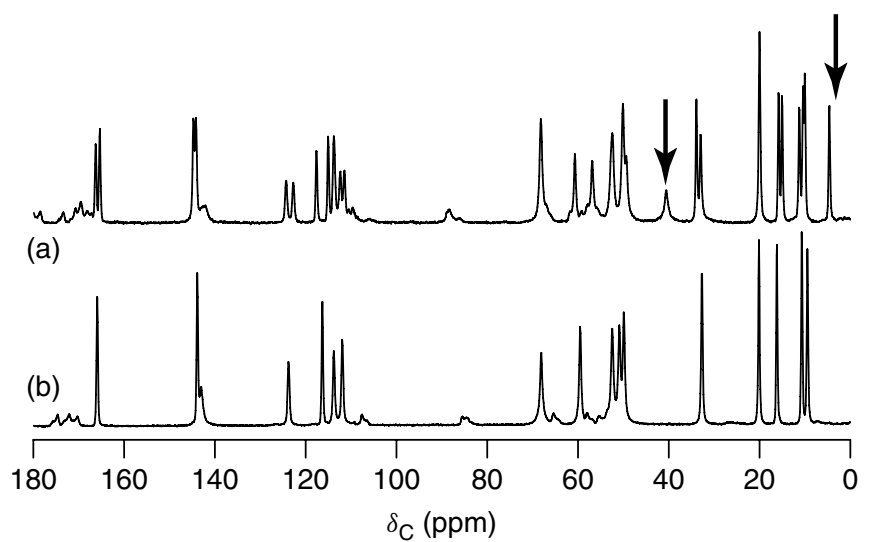

Figure 5 Carbon-13 CPMAS spectra of oxybuprocaine hydrochloride (III) form I (b) and form II (a), with arrows indicating the unusual positions for an ethyl group in the latter

solution and crystalline states since conformational flexibility is normally inhibited in most solids. Solid-state NMR chemical shifts provide direct conformational information, as illustrated by the case of form II of oxybuprocaine hydrochloride (III). ${ }^{16}$ The ${ }^{13} \mathrm{C}$ CPMAS spectrum of this compound (Figure 5) shows the existence of signals at unusual shifts for an ethyl group, 4-7 ppm to low frequency of those for the solution state. These are consistent with the expected positions for a gauche-gauche conformation (influenced by the well-known " $\gamma$-gauche shielding effect"). 
<smiles>CCCCOc1cc(C(=O)OCC[NH+](CC)CC)ccc1N</smiles>

Dipolar coupling, being through space, can also give information on conformations - in principle more directly than "scalar" coupling can. Thus, Terao and coworkers ${ }^{17}$ were able to determine the complete molecular structure of glycylisoleucine (IV), involving six dihedral angles, via selective dipolar recoupling experiments (see Three-Dimensional Molecular Structures in Uniformly Labelled Solids Determined Using Rotational Resonance in the Tilted Rotating Frame). Chemical shifts and dipolar coupling constants are being increasingly used to determine the secondary structure of crystalline (and amorphous) proteins ${ }^{18}$ (see NMR in Biochemistry: Multidimensional Spectroscopy for Structure Determination of Proteins in the Solid State; Structure Determination of Solid Proteins Using MAS and Isotopic Enrichment).<smiles>CCC(C)C(NC(=O)O)C(=O)O</smiles>

A second key aspect of crystallography concerns intermolecular interactions and the intermolecular environment. Here also, NMR has a strong role to play (see Intermolecular Interactions in Crystalline Solids). One common stabilizing influence for crystal structures is the existence of intermolecular hydrogen bonding. The presence of such bonding and its location can frequently be attested by the chemical shift of a relevant nucleus (see Hydrogen Bonding in Crystalline Organic Solids). Thus, for form III of cortisone acetate $(\mathbf{V})$, which has three molecules in the asymmetric unit, isotropic ${ }^{13} \mathrm{C}$ chemical shifts (assigned via measurements of the anisotropies and asymmetries) show ${ }^{\mathbf{1 9}}$ that two such molecules have hydrogen bonds involving the carbonyl group at position $\mathrm{C} 3$, while the third bonds through the carbonyl group at position $\mathrm{C} 22$. Intermolecular hydrogen bonding $(\mathrm{NH}-\mathrm{N})$ has also been detected by establishing the existence of indirect spin-spin $\left(J_{\mathrm{NN}}\right)$ coupling. ${ }^{20}$

Additional information on atomic environments can come from the use of the tensorial nature of shielding (see Chemical Shift Anisotropy and Asymmetry: Relationships to Crystal Structure). For example, experimental measurements of the anisotropy and asymmetry of ${ }^{199} \mathrm{Hg}$ chemical shifts allow the coordination of mercury in its crystalline compounds to be deduced from simple theoretical considerations. ${ }^{21}$ Thus, Figure 6 shows the variation of bandshape expected as the local environment varies between linear $\mathrm{HgX}_{2}$ and trigonal $\mathrm{HgX}_{3}$. The compound $\left[\mathrm{Hg}\left(\mathrm{S}-2,3,5,6-\mathrm{Me}_{4} \mathrm{C}_{6} \mathrm{H}\right)_{3}\right]^{-}$illustrates this point: the measured values of the anisotropy and asymmetry of the ${ }^{199} \mathrm{Hg}$ chemical shift tensor indicate that the compound contains a distorted $\mathrm{HgS}_{3}$ environment.

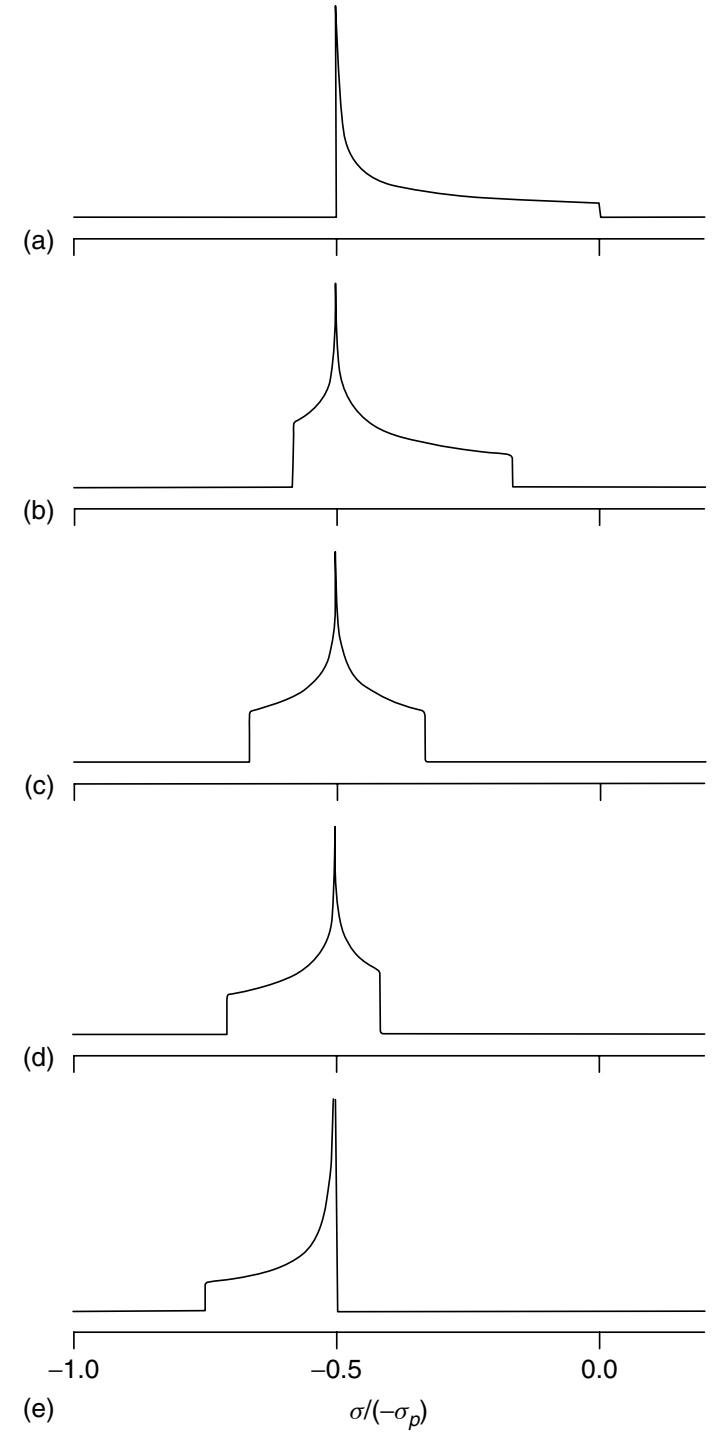

Figure 6 Schematic solid-state ${ }^{199} \mathrm{Hg}$ spectra for static samples, illustrating the effects of changes in mercury coordination from linear $\mathrm{HgX}_{2}$ (top) to trigonal $\mathrm{HgX}_{3}$ (bottom). (Reproduced with permission from Ref. 21, (C) 1997, Elsevier Science B.V.)

The multidimensional capability of NMR conveys considerable advantages and can reveal intermolecular effects. This is well seen for the simple case of methanol. ${ }^{22}$ Two-dimensional spectra involving chemical shift anisotropy and spin diffusion for ${ }^{13} \mathrm{C}$-enriched samples detect (Figure 7) differences in orientation between neighboring molecules because the spin-exchange process results in changes in the direction of the chemical shift principal axes. Moreover, there is a clear distinction between the $\alpha$ and $\beta$ forms of methanol, which is not readily apparent in the one-dimensional spectra. The shape of the band for the $\beta$ form in Figure 7(c) is simulated with the assumption that there is a $12^{\circ}$ angle between $\mathrm{C}-\mathrm{O}$ bond vectors in adjacent molecules. The simulated spectrum for the $\alpha$ form utilizes the crystal structure of Torrie et al. ${ }^{\mathbf{2 3}}$ It may be noted that the spectrum of the glassy sample shows rather random relative orientations of molecules in this case. ${ }^{\mathrm{c}}$ 

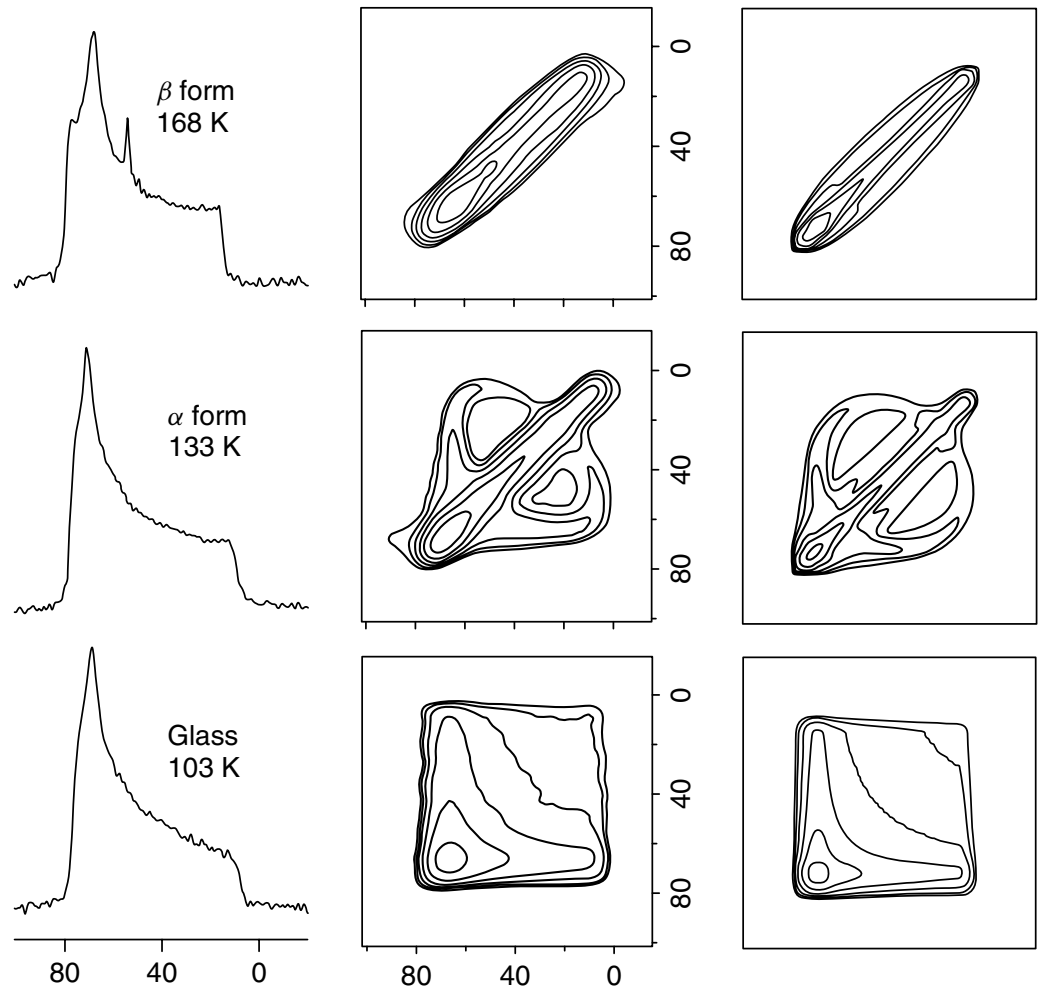

(a)

(b) ppm

(c)

Figure 7 Carbon-13 spectra of static samples for three forms of methanol: (a) one-dimensional spectra; (b) experimental EXSY spectra (involving spin diffusion during the mixing time); and (c) simulated spectra

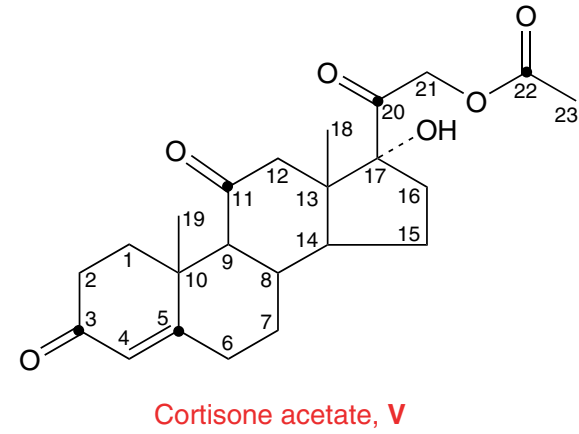

Isotropic chemical shifts give immediate information about host-guest systems, including solvates. This includes recognition of the nature of the guest molecules (which may not be immediately apparent from diffraction patterns if the guests are highly mobile ${ }^{\mathbf{8}}$. The relative positions of guest and host can also be addressed by studying the mutual effects on chemical shifts. In the case of incommensurate systems, which require considerations of $n$-dimensional ( $n>3)$ "superspace" for diffraction analysis, NMR can again provide immediate information on the guest molecules and on the host molecules separately.

Dipolar coupling is, in principle, a prime source of intermolecular environment information (see Dipolar and Indirect Coupling: Basics). For cases of hydrogen bonding, good results can be obtained. For instance, for L-tyrosine hydrochloride an intermolecular $\mathrm{C}-\mathrm{O}-\mathrm{H}$ distance of $2.47 \pm 0.07 \AA$ was obtained by NMR, ${ }^{24}$ which may be compared with the value $2.521 \AA$ reported from neutron diffraction experiments.
A truly intermolecular distance of $<3 \AA$ between aromatic protons has been found from double-quantum MAS work on a benzoxazine dimer. ${ }^{25} \mathrm{~N}, \mathrm{C}$ dipolar couplings have been used ${ }^{\mathbf{2 6}}$ to show the proximity of the $\alpha$-helix of one molecule of a 56 -amino acid protein to the $\mathrm{N}$ terminus of another molecule. Distances between carbon atoms in different fibrils of amyloid peptides have been explored by NMR. ${ }^{27}$ However, in general, there are considerable difficulties in quantifying intermolecular internuclear distances via measurements of dipolar coupling because multiple interactions frequently complicate the evaluations, even in the case of selective isotopic enrichment. This means that diffraction-derived structures are usually required to understand the NMR results.

A third key element of crystallography, which is imperfectly addressed by diffraction techniques, is molecular-level mobility. NMR has many approaches to this problem (see Intramolecular Motion in Crystalline Organic Solids; Rotational and Translational Dynamics), but a couple of examples will suffice at this point. In urea- and thiourea-inclusion compounds, the guest molecules are highly mobile, though in restricted ways. For the particular case of fluorocyclohexane as a guest, ring inversion is not greatly hindered by the channels, and variable-temperature ${ }^{19} \mathrm{~F}$ spectra reveal ${ }^{28}$ a classic coalescence phenomenon (Figure 8), from which the activation thermodynamic parameters can be derived.

Mobility information can come from quadrupolar spectra as well as those of spin- $\frac{1}{2}$ nuclides. Thus, molecular-level mobility can, in some circumstances, affect the linewidths of isotropic STMAS spectra but not isotropic MQMAS spectra $^{29}$ (Figure 9). Deuterium bandshapes of static samples are powerful indicators of mobility (see Deuterium NMR in 


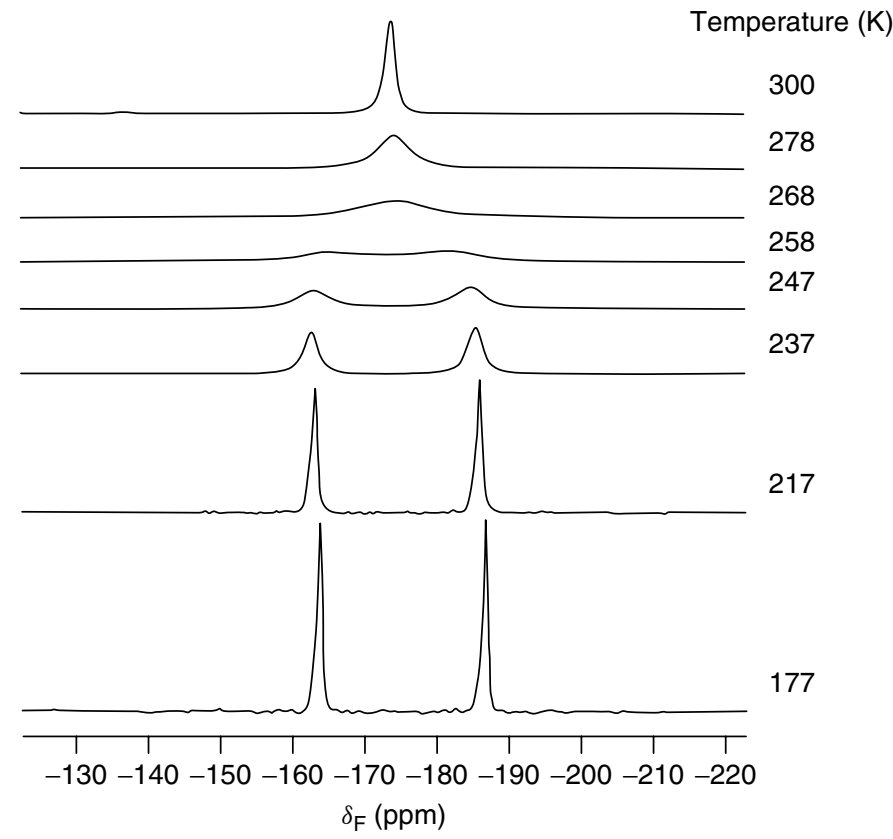

Figure 8 Experimental ${ }^{19} \mathrm{~F}$ MAS spectra (with proton decoupling) of the fluorocyclohexane/thiourea inclusion compound for various temperatures, showing the coalescence of the axial and equatorial signals resulting from ring inversion

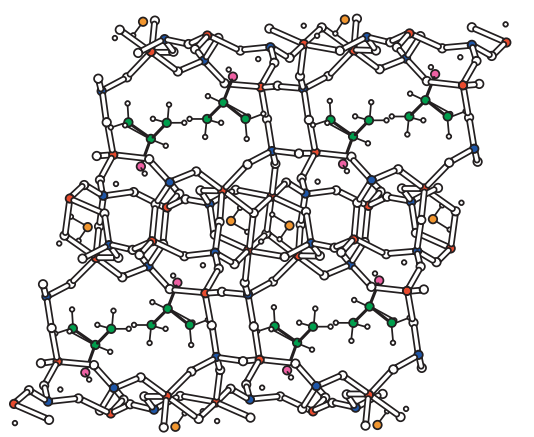

(a)
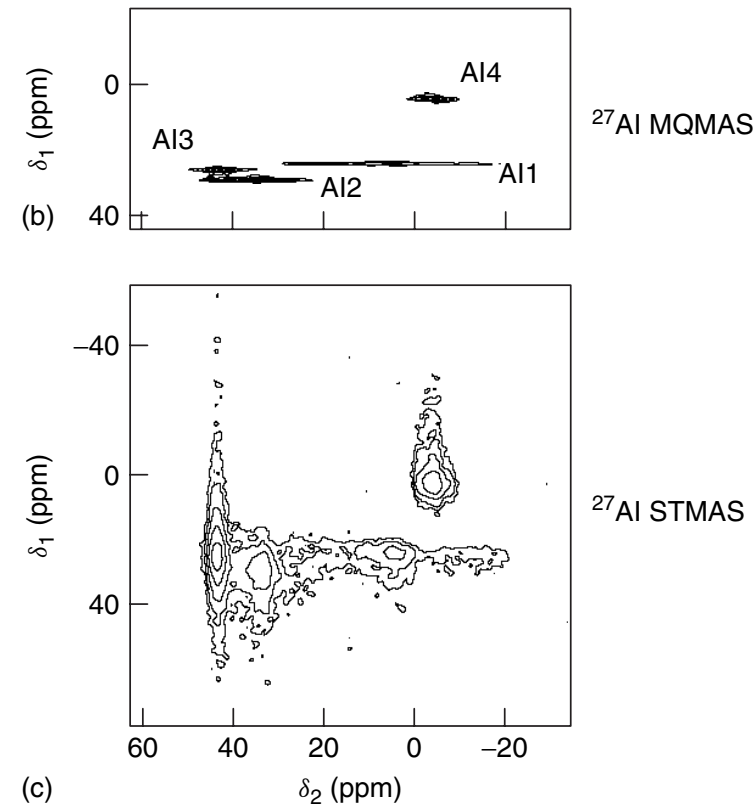

${ }^{27}$ AI STMAS

Figure 9 Structure of isopropylamine-ALPO-14 (a) and its two-dimensional ${ }^{27} \mathrm{Al}$ triple-quantum MQMAS (b) and STMAS (c) spectra, with the linewidths for the latter showing evidence of complex molecular mobility of the host:guest system

Solids). For instance, they have been used $^{\mathbf{3 0}}$ to accurately describe the nature of the motion of guest benzene and pyridine molecules within calixarene inclusion compounds. Note that, as in many cases, diffraction-derived structural information formed an important background to the investigation, but that the "added value" of NMR was essential to delineate the motional effects.

As already mentioned, relaxation times form another fruitful source of mobility information, extending down to lifetimes in the microseconds region.

\subsection{Crystal Structure Information}

The previous section has specifically dealt with chemical aspects of crystallography. However, NMR also provides detailed information of the more classical symmetry aspects of the subject.

Diffraction patterns are sometimes complicated by the existence of several independent molecules of the same species in the unit cell. Thus, information on the size of the asymmetric unit is valuable in advance of diffraction experiments. NMR is 


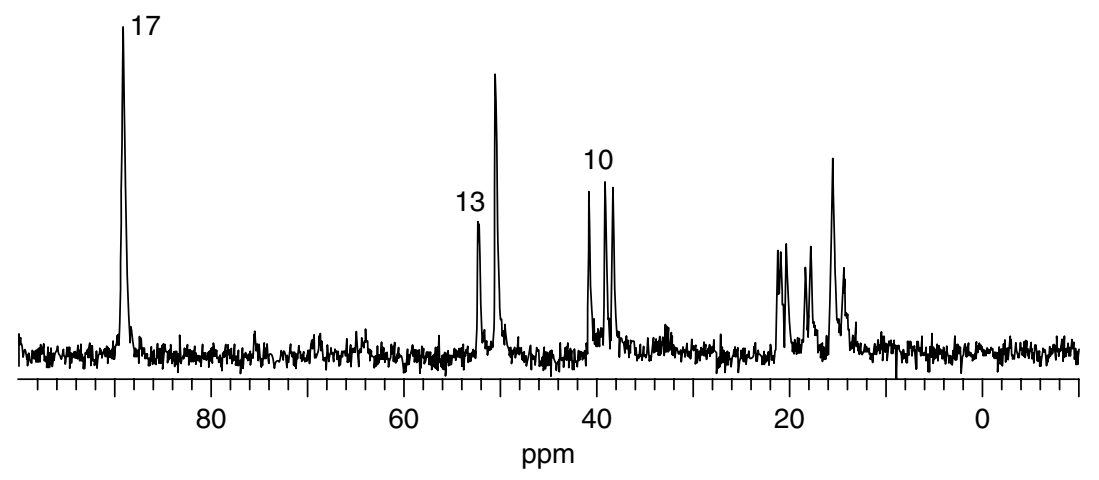

Figure 10 Portion of the dipolar dephased ${ }^{13} \mathrm{C}$ CPMAS spectrum of cortisone acetate $(\mathbf{V})$, form III, showing the resonance of C10 split into three because there are three molecules in the asymmetric unit. Note that $\mathrm{C} 13$ only appears as a 1:2 doublet and the three components for $\mathrm{C} 17$ are not resolved. (Reproduced with permission from Ref. 31, (c) 1990, Elsevier Ltd.)
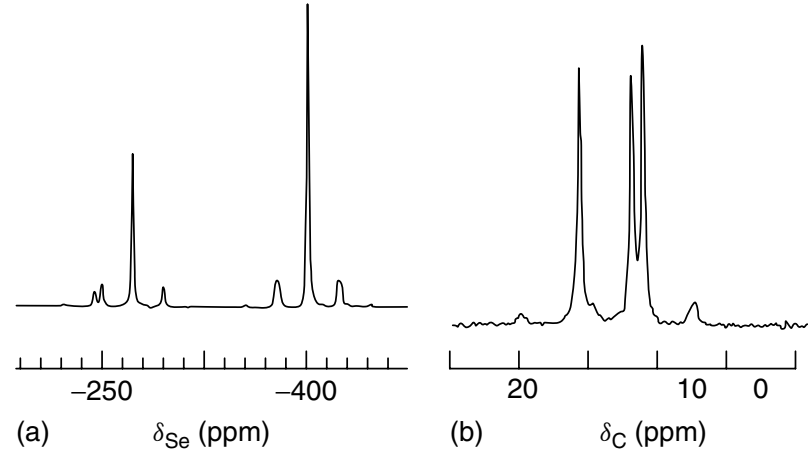

Figure 11 (a) Selenium-77 MAS spectrum of $\left(\mathrm{Me}_{2} \mathrm{SnSe}\right)_{3}$, showing that there is twofold but not threefold molecular symmetry and (b) carbon-13 spectrum of $\left(\mathrm{Me}_{2} \mathrm{SnS}\right)_{3}$, showing that the twofold symmetry is that of a rotation axis and not of a mirror plane. The small signals are either $J_{\mathrm{SnSe}}$ satellites or sinning sidebands

ideal for such determinations, since if there are, for example, two molecules in the asymmetric unit, then in principle all resonances will be split into two (though, of course, resolution may be inadequate in some cases) - a fact that is immediately recognizable. The case of form III of cortisone acetate has already been mentioned above. Figure 10 shows $^{\mathbf{3 1}}$ the clear splitting for the resonance of C10 into three equalintensity peaks. Of course, asymmetric units can be smaller than a complete molecule, as is the case for the trinuclear dimethyl chalcogenides, $\left(\mathrm{Me}_{2} \mathrm{SnE}\right)_{3}, \mathrm{E}=\mathrm{S}, \mathrm{Se} .^{32}$ In this case, observation of a $2: 1$ or $1: 2$ doublet for ${ }^{119} \mathrm{Sn}$ (and ${ }^{77} \mathrm{Se}$ ) shows that the asymmetric unit is half a molecule. However, additional crystallographic information is obtained by making use of the multinuclear nature of NMR; thus, observation of a 1:1:1 triplet for ${ }^{13} \mathrm{C}$ shows that the molecule has a $C_{2}$ symmetry axis rather than a mirror plane (Figure 11). NMR information on the content of the asymmetric unit is also of importance for understanding the crystal structure of network systems (see Polymorphism and Related Phenomena), as is the case for silicon carbide. This compound exists in a number of forms, known as polytypoids, which are distinguished by different modes of repetition of planes of atoms. Figure 12 shows the ${ }^{29} \mathrm{Si}$ spectra ${ }^{33}$ of four such forms, two of which have only one pair of $\mathrm{SiC}$ atoms in the asymmetric unit, whereas the other two have two and three such pairs, respectively.
It should be stressed that the nature of NMR equivalence between nuclear sites needs to be understood (see Spin Systems and Interactions in Solid-State NMR). When two positions are related by a center of symmetry, then they are fully equivalent as far as mutual isotropic $(J)$ coupling is concerned, and a single resonance is to be expected under MAS conditions (unless there is indirect coupling to other nuclei), in spite of such coupling. A single line is also expected under fast MAS conditions even when dipolar coupling (or anisotropic $J$ ) is involved. However, for static samples, the nuclei will still be subject to splittings arising from dipolar coupling (depending on the orientation of the internuclear vector with respect to the applied magnetic field), and this continues to be true under slow MAS conditions, thus leading to complex spectra. On the other hand, nuclei related by rotation axes or mirror planes will not, in general, be equivalent as far as isotropic $J$-coupling is concerned under static or slow MAS conditions, and dipolar coupling will even affect the spectra under fast MAS.

Quadrupole coupling tensors provide direct information on the site symmetry of atoms in crystal structures. Table 1 lists the EFG parameters of a central atom, M, for various coordination sites, as derived from a point-charge approximation. ${ }^{\mathbf{3 4}}$ The parameter $A$ is given in Reference 29. As for spectra of spin- $\frac{1}{2}$ nuclei, immediate information on the asymmetric unit can be obtained for quadrupolar nuclei. Thus, for (CO) ${ }_{5} \mathrm{MnPbPh}_{3}$ (of unknown crystal structure) ${ }^{55} \mathrm{Mn}$ MQMAS spectra at high magnetic field ${ }^{\mathbf{3 5}}$ (Figure 13) show that there are four independent molecules present, with slightly different quadrupolar parameters.

Table 1 Electric Field Gradient Parameters for a Central Atom with Various Coordinations

\begin{tabular}{lccc}
\hline Complex & Symmetry & EFG & $\eta_{Q}$ \\
\hline $\mathrm{MX}_{4}$ & $T_{d}$ & 0 & 0 \\
$\mathrm{MX}_{3} \mathrm{Y}$ & $C_{3 v}$ & $-2 A$ & 0 \\
$\mathrm{MX}_{2} \mathrm{Y}_{2}$ & $C_{2 v}$ & $\pm 2 A$ & 1 \\
$\mathrm{MX}_{6}$ & $O_{h}$ & 0 & 0 \\
$\mathrm{MX}_{5} \mathrm{Y}$ & $C_{4 v}$ & $-2 A$ & 0 \\
trans $-\mathrm{MX}_{4} \mathrm{Y}_{2}$ & $D_{4 h}$ & $-4 A$ & 0 \\
cis $-\mathrm{MX}_{4} \mathrm{Y}_{2}$ & $C_{2 v}$ & $2 A$ & 0 \\
mer $-\mathrm{MX}_{3} \mathrm{Y}_{3}$ & $C_{2 v}$ & $\pm 3 A$ & 1 \\
Fac $-\mathrm{MX}_{3} \mathrm{Y}_{3}$ & $C_{3 v}$ & 0 & 0 \\
\hline
\end{tabular}




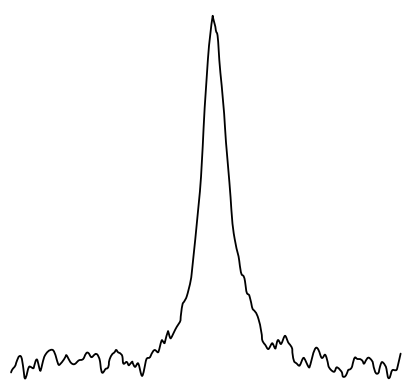

(a)

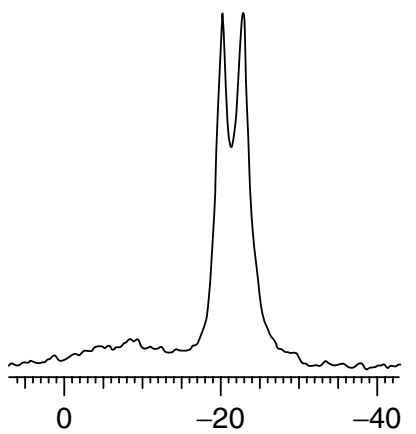

(c)

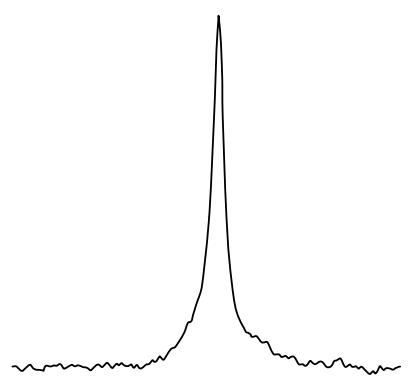

(b)

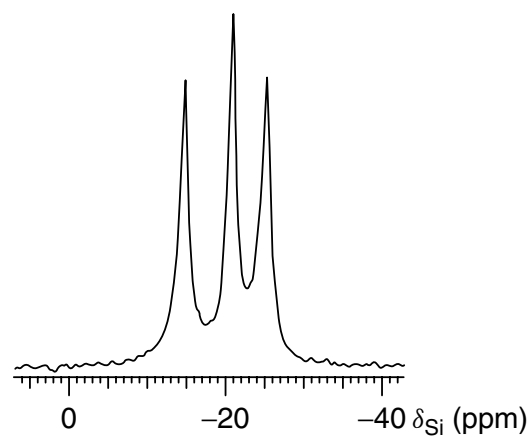

(d)

Figure 12 Silicon-29 MAS NMR spectra of four polytypes of silicon carbide, showing the differing numbers of silicon atoms in the asymmetric unit: (a) $3 \mathrm{C}$; (b) $2 \mathrm{H}$; (c) $4 \mathrm{H}$; and (d) $6 \mathrm{H}$

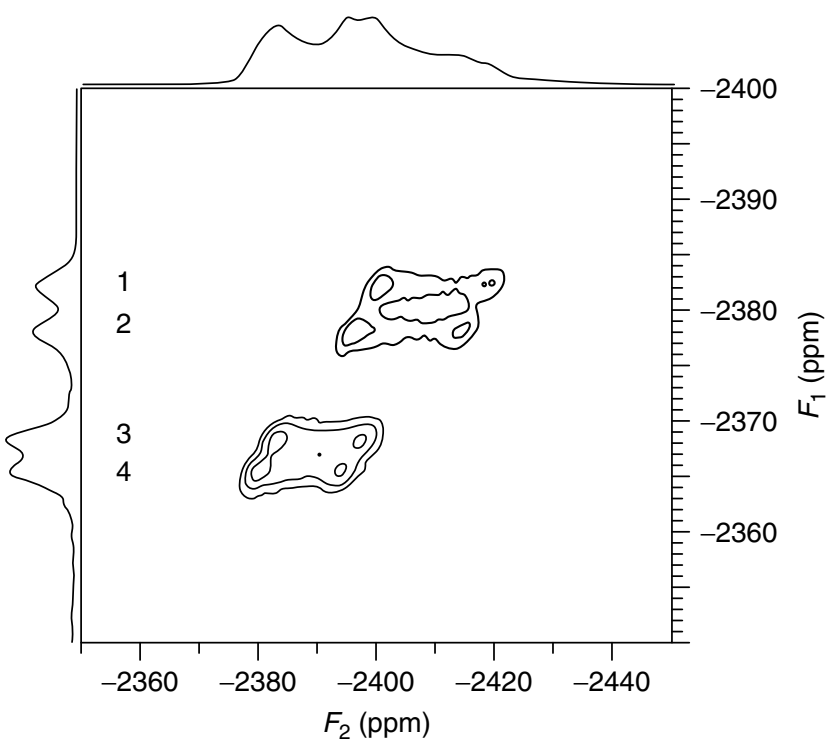

Figure 13 Manganese-55 two-dimensional triple-quantum MAS spectrum of $(\mathrm{CO})_{5} \mathrm{MnPbPh}_{3}$, clearly showing that there are four nonequivalent sites for the molecule. (Reproduced with permission from Ref. 35, (c) 2006, American Chemical Society)

Interplay between different NMR tensors (e.g., between dipolar and shielding tensors) can supply additional information, such as the relative orientations of the two tensors, even from powder NMR experiments (see Tensor Interplay). This is particularly powerful if the orientation of one of those tensors in the crystal structure is known or can be deduced, as is the case for the dipolar coupling between directly bonded atoms.
There are various types of crystal structures that depart from the simple ideal of three-dimensional repetition. These include nonstoichiometric systems, defect structures, disordered structures, and incommensurate compounds. For all these cases, NMR can supply information that is not available from diffraction measurements (see Inorganic Non-Stoichiometric Crystalline Systems). For instance, it can sometimes distinguish between spatial and temporal disorder by virtue of the different timescales available to the two techniques. This matter is further explored in Intramolecular Motion in Crystalline Organic Solids, but one example here makes the point. The azobenzene dyestuff DR278 was shown by single-crystal diffraction techniques to contain the molecules in two different conformations (with unequal occupancies) in the unit cell. NMR demonstrated ${ }^{\mathbf{3 6}}$ unequivocally that there was exchange between the two forms (temporal disorder), and a combination of selective population inversion and bandshape analysis enabled the thermodynamic parameters for the exchange to be derived. Additionally, two-dimensional work provided the relative orientations of ${ }^{15} \mathrm{~N}$ shielding principal axes in the two forms (consistent with the diffraction data).

Furthermore, where X-ray diffraction struggles to recognize the differences between neighboring atoms in the periodic table (especially when isoelectronic), NMR has no such problem; this is one aspect where the multinuclear character of NMR conveys a considerable advantage. It is particularly important for ceramic materials and zeolites because of the electronic similarity of oxygen and nitrogen and of the $\mathrm{SiN}$ and $\mathrm{AlO}$ pairs of atoms. Thus, ${ }^{15} \mathrm{~N}$ NMR has been used ${ }^{37}$ to determine the ordering of anionic elements (nitrogen and oxygen) in $J$-phase yttrium silicon oxynitride, $\mathrm{Y}_{4} \mathrm{Si}_{2} \mathrm{O}_{7} \mathrm{~N}_{2}$.

Simple NMR information on the number of atoms, ions, or molecules in the asymmetric unit can sometimes leads to 


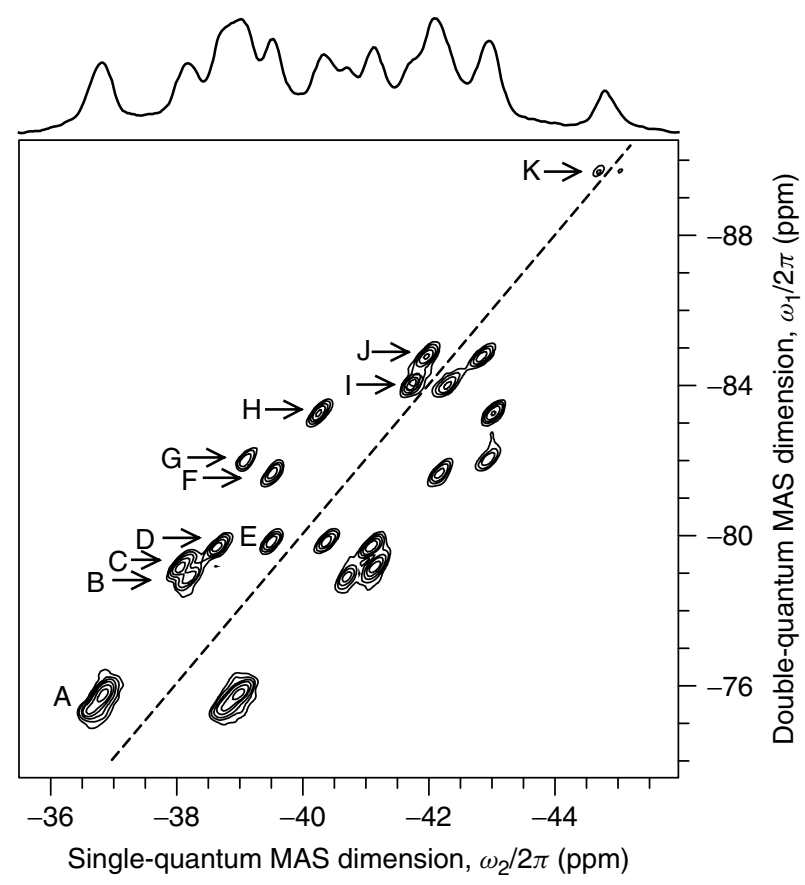

Figure 14 Phosphorus-31 two-dimensional through-bond doublequantum MAS correlation spectrum of $\mathrm{ZrP}_{2} \mathrm{O}_{7}$ obtained at $12-\mathrm{kHz}$ spinning frequency using the refocused INADEQUATE experiment. The dashed line indicates the diagonal of the spectrum. The onedimensional MAS spectrum is shown above the two-dimensional plot

evaluation of the space group. Thus, the through-bonds doublequantum MAS correlation ${ }^{31} \mathrm{P}$ spectrum of $\mathrm{ZrP}_{2} \mathrm{O}_{7}$ (Figure 14) shows ${ }^{38}$ that the unit cell contains 13 unsymmetrical $\mathrm{P}_{2} \mathrm{O}_{7}$ units and the corresponding through-space case indicates that, in addition, there is one symmetrical $\mathrm{P}_{2} \mathrm{O}_{7}$ unit. This is consistent with a $\mathrm{Pbca}$ space group and not with the $P a \overline{3}$ structure, which had been assumed earlier. The NMR information allowed the structure to be obtained from powder XRD data.

\section{CONCLUDING REMARKS}

Interplay between diffraction and solid-state NMR, as mentioned above, is now common. It usually consists merely of the NMR data being interpreted in terms of the structure determined by diffraction or by the two techniques being looked at in parallel, rather than seeing the information content gain by synergy. However, the future would seem to hold better promise. It has been demonstrated that structure solutions can be obtained by constraining the possible solutions to powder diffraction analysis according to NMR information, using a computer protocol that integrates the two types of information. The structure of form II of cortisone acetate has been obtained in this way, ${ }^{\mathbf{1 0}}$ even though only a single piece of (semiquantitative) NMR information was included in the input. An analogous combination of NMR information, powder diffraction data, and computing methods has proved successful in determining the crystal structure of $N$-( $p$-tolyl)dodecylsulfonamide. ${ }^{39}$ Dipolar coupling results can form even more powerful constraints on crystal structures. ${ }^{\mathbf{4 0}}$ The ultimate goal may be to incorporate such computations into procedures to determine crystal structures by the simultaneous refinement of NMR and diffraction data.

In parallel with such developments, other advances are being made in the computation area. Specifically, shielding, indirect coupling, and quadrupolar coupling tensors can now be computed for solids by taking account of the repetition inherent in crystal structures (see Computations of Magnetic Resonance Parameters for Crystalline Systems: Principles). The planewave-pseudopotential approach, as used in the CASTEP protocol, ${ }^{41}$ is increasingly applied (see Chemical Shift Computations for Crystalline Molecular Systems: Applications). This and other computational developments are revealing new understanding of the factors affecting NMR parameters in solids.

As mentioned earlier, it has now been possible to solve a complete crystal structure using NMR data 4 (together with unit cell parameters and the space group from a powder diffraction study). In this case, interatomic distances were obtained by ${ }^{29} \mathrm{Si}$ double-quantum dipolar recoupling experiments. An approach using NMR criteria to decide between computer-generated crystal structures (selected on the basis of lattice energy computations) has shown to have considerable potential for determining structures without diffraction input. ${ }^{42}$ Significantly, this work made use of the full chemical shift tensor information. NMR crystallography imposes considerable constraints on allowed space groups in many cases, leading to full structure determination ${ }^{\mathbf{4 3}}$ (see Crystallography by NMR: Fundamental Concepts). It has also been shown that in certain circumstances NMR data can reveal information more accurately than that by diffraction analysis. Thus, Facelli and Grant ${ }^{44}$ used ${ }^{13} \mathrm{C}$ shielding tensors to prove that there was a lack of symmetry between atoms in naphthalene crystals, which accorded with the known space group, whereas the geometry as determined by the diffraction experiments suggested symmetry within experimental error. A combination of experimental chemical shift tensors and shielding computations has been used to improve the crystal structure of a zeolite obtained from powder XRD data, creating an "NMR crystallography structure refinement strategy".45 Of course, these examples are unusual cases and NMR will certainly never replace diffraction techniques for the determination of crystal structures. However, it is equally certain that the importance of NMR to crystallography will grow as its ability to provide essential complementary information is increasingly recognized. The subject of NMR crystallography is thriving and is set to make further substantial contributions to our understanding of the nature of the crystalline state.

\section{RELATED ARTICLES}

Accuracy Limitations on Internuclear Distances Measured by REDOR; Dipolar and Indirect Coupling Tensors in Solids; Dynamics in Solid Organic Compounds: Intramolecular Motions; High Temperature Superconductors; Inclusion Compounds; Incommensurate Systems; Intercalation Compounds; Molecular Sieves: Crystalline Systems; Reorientation in Crystalline Solids: Propeller-Like $\mathrm{R}_{3} \mathrm{M}$ Species; Silicon-29 NMR of Solid Silicates; Spinning Sideband Analysis for Spin-1/2 Nuclei; Through-Bond Experiments in Solids; Ultraslow Motions in Solids. 


\section{END NOTES}

a One referee objected to the term NMR crystallography in the title of a submitted paper as follows:

- Since the authors are dealing with powders rather than crystals, the use of the term crystallography is inappropriate.

- Crystallography specifically applies to diffraction of electromagnetic radiation.

Neither comment appears apposite!

b In principle, indirect coupling is a tensor property, but its anisotropy and asymmetry have so far proved to be of limited use.

c While amorphous systems can scarcely come under the heading of "crystallography" and are not otherwise dealt with in this article, this example is included here to emphasize that NMR is not limited to crystalline systems.

\section{REFERENCES}

1. A. Isaacs, J. Daintith and E. Martin, eds, Oxford Dictionary of Science, $4^{\text {th }}$ edn., Oxford University Press, ISBN 0192800981, 1999.

2. G. E. Pake, J. Chem. Phys., 1948, 16, 327-336.

3. H. S. Gutowsky, G. B. Kistiakowsky, G. E. Pake, and E. M. Purcell, J. Chem. Phys., 1949, 17, 972-981.

4. D. H. Brouwer, R. J. Darton, R. E. Morris, and M. H. Levitt, J. Am. Chem. Soc., 2005, 127, 10365-10370.

5. S. G. Zech, A. J. Wand, and A. E. McDermott, J. Am. Chem. Soc., 2005, 127, 8618-8626.

6. M. A. Kennedy and P. D. Ellis, Concepts Magn. Reson., 1989, 1, $35-47$ and $109-129$.

7. R. K. Harris, Analyst, 2006, 131, 351-373.

8. A. Othman, J. S. O. Evans, I. R. Evans, R. K. Harris, and P. Hodgkinson, J. Pharm. Sci., 2007, 96, 1380-1396.

9. K. D. M. Harris, M. Tremayne, and B. M. Kariuki, Angew. Chem. Int. Ed., 2001, 40, 1626-1651.

10. R. K. Harris, Solid State Sci., 2004, 6, 1025-1037.

11. T. N. Pham, S. Masiero, G. Gottarelli, and S. P. Brown, J. Am. Chem. Soc., 2005, 127, 16018-16019.

12. H. W. Kroto, S. L. Klein, M. F. Meidine, J. F. Nixon, R. K. Harris, K. J. Packer, and P. Reams, J. Organomet. Chem., 1985, 280, 281-287.

13. C. A. Fyfe, H. Gies, and Y. Feng, J. Am. Chem. Soc., 1989, 111, $7702-7707$.

14. A. Lesage, C. Auger, S. Caldarelli, and L. Emsley, J. Am. Chem. Soc., 1997, 119, 7867-7868.

15. V. Munch, F. Taulelle, T. Loiseau, G. Férey, A. K. Cheetham, S. Weigel, and G. D. Stucky, Magn. Reson. Chem., 1999, 37, S100-S107.

16. R. K. Harris, S. Cadars, L. Emsley, J. R. Yates, C. J. Pickard, R. K. R. Jetti, and U. J. Griesser, Phys. Chem. Chem. Phys., 2007, 9, 360-368

17. K. Nomura, K. Takegoshi, T. Terao, K. Uchida, and M. Kainosho, J. Am. Chem. Soc., 1999, 121, 4064-4065.

18. A. E. McDermott, Curr. Opin. Struct. Biol., 2004, 14, 554-561.

19. E. A. Christopher, R. K. Harris, and R. A. Fletton, Solid State NMR, 1992, 1, 93-101.
20. T. N. Pham, J. M. Griffin, S. Masiero, S. Lena, G. Gottarelli, P. Hodgkinson, C. Filip, and S. P. Brown, Phys. Chem. Chem. Phys., 2007, 9, 3416-3423.

21. G. A. Bowmaker, R. K. Harris, and S.-W. Oh, Coord. Chem. Rev., 1997, 167, 49-94.

22. R. Tycko and G. Dabbagh, J. Am. Chem. Soc., 1991, 113, 3592-3593.

23. B. H. Torrie, S.-X. Weng, and B. M. Powell, Mol. Phys., 1989, 67, $575-581$

24. B.-J. van Rossum, C. P. de Groot, V. Ladizhansky, S. Vega, and H. J. M. de Groot, J. Am. Chem. Soc., 2000, 122, 3465-3472.

25. I. Schnell, S. P. Brown, H. Y. Low, H. Ishida, and H. W. Spiess, J. Am. Chem. Soc., 1998, 120, 11784-11795.

26. J. J. Helmus, P. S. Nadaud, N. Höfer, and C. P. Jaroniec, J. Chem. Phys., 2008, 128, article no. 052314.

27. R. Tycko, J. Chem. Phys., 2007, 126, article no. 064506.

28. R. K. Harris, A. Nordon, and K. D. M. Harris, Magn. Reson. Chem., 1999, 37, 15-24.

29. S. Antonijevic, S. E. Ashbrook, S. Biedasek, R. I. Walton, S. Wimperis, and H. Yang, J. Am. Chem. Soc., 2006, 128, 8054-8062.

30. E. B. Brouwer, G. D. Enright, C. I. Ratcliffe, G. A. Facey, and J. A. Ripmeester, J. Phys. Chem. B, 1999, 103, 10604-10616.

31. R. K. Harris, A. M. Kenwright, B. J. Say, R. R. Yeung, R. A. Fletton, R. W. Lancaster, and G. L. Hardgrove, Spectrochim. Acta, 1990, 46A, 927-935.

32. R. K. Harris, and A. Sebald, Magn. Reson. Chem., 1989, 27, $81-87$.

33. D. C. Apperley, R. K. Harris, G. L. Marshall, and D. P. Thompson, J. Am. Ceram. Soc., 1991, 74, 777-782.

34. O. H. Han and E. Oldfield, Inorg. Chem., 1990, 29, 3666-3669.

35. K. J. Ooms, K. W. Feindel, V. V. Terskikh, and R. E. Wasylishen, Inorg. Chem., 2006, 45, 8492-8499.

36. G. McGeorge, R. K. Harris, A. S. Batsanov, A. M. Chippendale, J. F. Bullock, and Z. Gan, J. Phys. Chem. A, 1998, 102, 3505-3513.

37. D. S. B. Hauck, R. K. Harris, D. C. Apperley, and D. P. Thompson, J. Mater. Chem., 1993, 3, 1005-1006.

38. I. J. King, F. Fayon, D. Massiot, R. K. Harris and J. S. O. Evans, Chem. Commun., 2001, 1766-1767.

39. M. Rajeswaran, T. N. Blanton, N. Zumbulyadis, D. J. Giesen, C. Conesa-Moratilla, S. T. Misture, P. W. Stephens, and A. Huq, J. Am. Chem. Soc., 2002, 124, 14450-14459.

40. D. A. Middleton, X. Peng, D. Saunders, K. Shankland, W. I. F. David, and A. J. Markvardsen, Chem. Comm., 2002, 1976-1977.

41. J. R. Yates, C. J. Pickard, and F. Mauri, Phys. Rev. B, 2007, 76, article no. 024401.

42. J. K. Harper and D. M. Grant, Cryst. Growth Des., 2006, 6, 2315-2321.

43. F. Taulelle, Solid State Sci., 2004, 6, 1053-1057.

44. J. C. Facelli and D. M. Grant, Nature, 1993, 365, 325.

45. D. H. Brouwer, J. Magn. Reson., 2008, 194, 136-146.

\section{Acknowledgments}

The author is very grateful to other authors of Encyclopedia, whose articles are related to this one, for their suggestions and, in some cases (Professors Wimperis, Tycko and Wasylishen), provision of figures. Some of the examples used herein are those from author's own research group. The author is highly indebted to the research students and colleagues involved in the work. 


\section{Biographical Sketch}

Robin K. Harris, b. 1936. B.A. (Nat. Sci.), Ph.D. (supervisor Norman Sheppard), Sc.D., University of Cambridge, UK. Postdoctoral work at Mellon Institute, Pittsburgh, PA (with Aksel Bothner-By). Successively lecturer, senior lecturer, and professor, University of East Anglia, UK.
Professor of Chemistry, University of Durham, UK, 1984-2002. Currently Emeritus Professor. Secretary-General of ISMAR, 1986-1992. Approx. 520 Publications, including a textbook "Nuclear Magnetic Resonance Spectroscopy: A Physicochemical View". Current research specialty: solid-state NMR, in particular, NMR crystallography and polymorphism. 